\title{
Generating a Higgs Potential Quartic Term
}

\author{
Csaba Csáki, ${ }^{1}$ Cong-Sen Guan $\odot,{ }^{1,2,3}$ Teng $\mathrm{Ma}^{2,4, \dagger}$ and Jing Shu $\odot^{2,3,5,6,7,8, *}$ \\ ${ }^{1}$ Department of Physics, LEPP, Cornell University, Ithaca, New York 14853, USA \\ ${ }^{2}$ CAS Key Laboratory of Theoretical Physics, Institute of Theoretical Physics, Chinese Academy of Sciences, Beijing 100190, China \\ ${ }^{3}$ School of Physical Sciences, University of Chinese Academy of Sciences, Beijing 100049, People's Republic of China \\ ${ }^{4}$ Physics Department, Technion, Israel Institute of Technology, Haifa 3200003, Israel \\ ${ }^{5}$ CAS Center for Excellence in Particle Physics, Beijing 100049, China \\ ${ }^{6}$ Center for High Energy Physics, Peking University, Beijing 100871, China \\ ${ }^{7}$ School of Fundamental Physics and Mathematical Sciences, Hangzhou Institute for Advanced Study, \\ University of Chinese Academy of Sciences, Hangzhou 310024, China \\ ${ }^{8}$ International Centre for Theoretical Physics Asia-Pacific, Beijing/Hangzhou, China
}

(Received 22 October 2019; revised manuscript received 2 June 2020; accepted 4 June 2020; published 24 June 2020)

\begin{abstract}
We present a simple mechanism for generating a Higgs quartic in composite Higgs models without a corresponding quadratic term. This quartic term will originate from a Higgs dependent kinetic mixing between additional fermionic states. The mechanism can be naturally embedded to models with maximal symmetry as well as twin Higgs models. The resulting twin Higgs models will have a fully natural realistic Higgs potential, where the quartic mechanism will serve as the only source for the $Z_{2}$ breaking, while the top and gauge sectors can remain exactly $Z_{2}$ invariant.
\end{abstract}

DOI: 10.1103/PhysRevLett.124.251801

The sensitivity of the Higgs potential to very large energy scales makes it unstable to quantum corrections. Stabilization of the scale of electroweak symmetry breaking (EWSB) is one of the most profound problems in particle physics. One can eliminate this sensitivity to UV scales by introducing new physics (such as supersymmetry) not too far above the EWSB scale. Of such new physics models, the pseudo-Nambu-Goldstone boson (PNGB) Higgs [1-5] (for reviews, see [6-9]) is one of the simplest and most widely studied. The mechanisms that ensure that the resulting Higgs potential remains free of UV divergences include collective symmetry breaking [4], discrete symmetry $[10,11]$, or maximal symmetry $[12,13]$.

In order to generate a potential for the PNGB Higgs, the global symmetry $G$ (whose spontaneous breaking gives rise to the PNGB) has to also be explicitly broken. The resulting Higgs potential can be parametrized as an expansion of some small (soft) explicit breaking. The main difficulty of such models is that generically only Higgs quadratic terms are generated at the leading order while both quadratic and quartic terms are generated at the next-to-leading order. On the other hand, the Higgs mass of $125 \mathrm{GeV}$ requires that the quartic be much less suppressed than the quadratic term. Hence, usually a tuning of the order of a few percent level is

Published by the American Physical Society under the terms of the Creative Commons Attribution 4.0 International license. Further distribution of this work must maintain attribution to the author(s) and the published article's title, journal citation, and DOI. Funded by SCOAP . needed to produce the little hierarchy of the EWSB and the new physics scales. Models that produce an adjustable Higgs quartic term without introducing a Higgs quadratic provide an elegant solution to the little hierarchy problem. An example of this type are 6D models [14] where a treelevel quartic can originate from the gauge boson components along the extra dimension, or the little Higgs models $[4,15]$ based on dimensional deconstruction of the $6 \mathrm{D}$ theory. However, these models are usually quite complicated and also require additional PNGBs, such as the second Higgs doublet (i.e., the generated quartic is that of a two-Higgs doublet model and not a true Standard Model (SM)-like Higgs quartic).

In this Letter we propose a novel mechanism to produce an adjustable Higgs quartic self-coupling from loop corrections without a corresponding Higgs quadratic term. We introduce an electroweak (EW) triplet and singlet fermion and observe that if their kinetic terms are independent of the Higgs field, we will only produce a Higgs quartic term in the one-loop effective potential but no Higgs quadratic term. The simple underlying reason is that a triplet-singlet mixing necessarily involves at least two Higgs insertions. Moreover, the sign of the generated quartic will be positive if the Yukawa term mixing the triplet and the singlet is momentum dependent (while the momentum independent mixing term always gives a negative contribution). The recently proposed maximal symmetry $[12,13]$ has exactly the right properties for this mechanism: its main effect is exactly to protect the effective kinetic terms from Higgs dependent corrections. Thus, models with maximal symmetry can naturally produce a positive and adjustable Higgs 
quartic term. Our mechanism can be simply implemented in any PNGB Higgs model based on deconstruction or warped extra dimensions scenario without having to introduce any additional structures. When we implement this mechanism in twin Higgs models, the additional quartic will allow the top and gauge sectors to remain exactly $Z_{2}$ invariant, thus fully natural EWSB without any tuning can be achieved.

First, we illuminate the essence of our simple mechanism for producing an adjustable Higgs quartic coupling based on PNGB Higgs model. We introduce the EW triplet $\Delta$ and singlet $\eta$ Dirac fermions, both of which are assumed to be elementary. For simplicity, first we assume that they are massless (but in the full model all allowed mass and Yukawa terms will be added). If the triplet and singlet mix through a Yukawa coupling involving the SM Higgs in the low energy effective theory, in momentum space the leading order Lagrangian will be given by

$$
\mathcal{L}=\operatorname{Tr}[\bar{\Delta} \not \not \Delta]+\bar{\eta} \not \not \eta-\left(\frac{\lambda}{f} H^{\dagger} \bar{\Delta} H \eta+\text { H.c. }\right),
$$

where $f$ will be the PNGB Higgs decay constant and $H$ is the Higgs doublet. The hypercharge of the two Dirac fermions are assumed to be zero. Note that the choice of triplet and singlet representations under $\mathrm{SU}(2)_{L}$ is essential: since $\Delta$ carries two $\mathrm{SU}(2)$ indices and $\eta$ does not carry any, their Yukawa coupling has to contain at least two Higgs doublets. Thus, if this mixing terms is the only Higgs dependent term in the effective Lagrangian (1) while the kinetic terms are Higgs independent, then one can only get a contribution to the Higgs quartic term without obtaining a shift in the Higgs mass term.

Treating the Higgs as a background field, it is easy to find that the leading contributions from the loop of the triplet and singlet fermions to the Higgs quartic selfcoupling is always negative:

$$
\begin{aligned}
V(H) & \sim-\frac{i}{2} \int \frac{d^{4} p}{(2 \pi)^{4}} \frac{\lambda^{2}\left(H^{\dagger} H\right)^{2}}{f^{2}}(-1) \operatorname{Tr}\left[\frac{i \not p}{p^{2}} \frac{i \not p}{p^{2}}\right] \\
& =-\frac{\lambda^{2}\left(H^{\dagger} H\right)^{2}}{f^{2}} \int \frac{d^{4} p_{E}}{(2 \pi)^{4}} \frac{2}{p_{E}^{2}},
\end{aligned}
$$

where in the second line we performed a Wick rotation to Euclidean space $p^{2} \rightarrow-p_{E}^{2}$, where $p_{E}^{2}=p_{0}^{2}+(\vec{p})^{2}$ is positive definite. From this examination of the correction it is, however, clear how this problem can be solved: we expect that if one uses a Higgs dependent kinetic mixing rather than a Yukawa mixing the sign could be reversed, since we will pick up an extra $p^{2}$ term in the Feynman diagram, which after Wick rotation will provide an additional sign flip $\left(p^{2} \rightarrow-p_{E}^{2}\right)$.

The Lagrangian describing the kinetic mixing of the triplet and singlet fermions can be parametrized as

$$
\mathcal{L}=\operatorname{Tr}[\bar{\Delta} \not p \Delta]+\bar{\eta} \not \not \eta-\left(\frac{\lambda}{f^{2}} H^{\dagger} \bar{\Delta} H \not p \eta+\text { H.c. }\right) .
$$

It is easy to check that the leading one-loop correction to the Higgs potential has an extra $p^{2}$ factor compared to the previous case, which will flip the sign of the contribution to the Higgs quartic after the Wick rotation to Euclidean space is performed. Thus, we find that for the case of kinetic mixing the induced Higgs quartic is always positive. We emphasize again that we have made the crucial assumption that the effective kinetic terms of the triplet and singlet fermions are Higgs independent. Otherwise corrections to the Higgs mass term will also be produced, and the quadratic and quartic terms would remain linked. Note that for the case of scalar triplet and singlet both the Yukawa and kinetic mixings will always produce a negative shift in the Higgs quartic. Hence, we can see that only the case of kinetic fermionic mixing will produce the desired positive shift in the Higgs quartic self coupling. To summarize, we found two necessary conditions for producing an adjustable and positive Higgs quartic in the triplet-singlet model: (i) The effective kinetic terms must be Higgs independent. (ii) The triplet-singlet mixing must be momentum dependent. The first condition is exactly the main consequence of models with maximal symmetry, which we will take advantage of.

Before we present our full model we would like to first demonstrate how such a kinetic mixing term can be easily generated in the effective theory. The key is to consider a chiral mixing between the fermions $\Delta, \eta$ and some heavy fermion $\psi$. Such linear couplings between elementary $(\Delta$, $\eta)$ and composite $(\psi)$ fields show up naturally in models of partial compositeness in composite Higgs models. For a simple illustration we introduce an $\mathrm{SU}(2)_{L}$ doublet fermion $\Psi_{2}$ with the most general chiral mixing with the $\Delta, \eta$ :

$\mathcal{L}_{\text {int }}=\lambda_{1 L} \bar{\Psi}_{2_{R}} \Delta_{L} H+\lambda_{2 L} \bar{\Psi}_{2_{R}} H \eta_{L}+(L \leftrightarrow R)+$ H.c.

After integrating out the heavy fermion we find the following effective mixing terms in the low-energy effective Lagrangian:

$$
\begin{aligned}
\mathcal{L}_{\text {eff }}^{\text {mix }}= & \frac{-1}{M^{2}-p^{2}}\left(\lambda_{1 L} \lambda_{2 L} H^{\dagger} \bar{\Delta}_{L} H \not p \eta_{L}\right. \\
& \left.+M \lambda_{1 L} \lambda_{2 R} H^{\dagger} \bar{\Delta}_{L} H \eta_{R}\right)+(L \leftrightarrow R)+\text { H.c., }
\end{aligned}
$$

where $M$ is the mass of the heavy field. We can see that in the general case we get both the kinetic and Yukawa mixings in the effective Lagrangian (leading to both positive and negative contributions to the Higgs quartic). However, one can easily turn off either the mass or the kinetic mixing by dialing the various $\lambda_{L, R}$ couplings. For example, if we turn off the right handed or the left handed couplings (e.g., $\lambda_{1,2 R}=0$ or $\lambda_{1,2 L}=0$ ) [16], we will only get the kinetic mixing term, while if we turn off one left 
handed and one right handed coupling (e.g., $\lambda_{1 R}=0$ and $\lambda_{2 L}=0$ ), we will only get the Yukawa mixing term. The simple lesson from this toy example is that a purely chiral mixing with the heavy fermion will produce the desired kinetic mixing in the effective theory.

Let us now show how to actually obtain a complete realistic model with all the essential ingredients explained above by embedding it into a simple two-site composite Higgs model. For this we will use the simplest implementation of maximal symmetry recently proposed in [13]. While the smallest implementation of minimal maximal symmetry is based on a $\mathrm{SO}(5) / \mathrm{SO}(4)$ coset space, we will instead focus on introducing the Higgs quartic mechanism into twin Higgs models [10,17-21] in this Letter, because we will obtain the most interesting realistic model with fully natural EWSB that way. Hence, we choose the $\mathrm{SO}(8) / \mathrm{SO}(7)$ coset space which is the minimal one to contain the complete $\mathrm{SM}+$ twin sector.

The setup of the two site $\mathrm{SO}(8) / \mathrm{SO}(7)$ coset space is similar to the $\mathrm{SO}(5) / \mathrm{SO}(4)$ case in [13], except one also needs to include the twin SM. Each site has its own $\mathrm{SO}(8)$ global symmetry, so we have an $\mathrm{SO}(8)_{1} \times \mathrm{SO}(8)_{2}$. The EW and its twin gauge symmetry are embedded in $\mathrm{SO}(8)_{1}$, while we assume that $\mathrm{SO}(8)_{2}$ is fully gauged. The link field $U_{1}$ in the bifundamental representation of the global symmetry breaks the global symmetry to the diagonal subgroup $\mathrm{SO}(8)_{V}$ and the scalar field $\mathcal{H}^{\prime}$ in the fundamental representation of $\mathrm{SO}(8)_{2}$ breaks the gauge symmetry $\mathrm{SO}(8)_{2}$ to $\mathrm{SO}(7)$. So the uneaten Nambu-Goldstone bosons (NGBs) are in the coset space $\mathrm{SO}(8)_{1} / \mathrm{SO}(7)$ as needed for twin Higgs models (THMs).

Based on the implementation of the two-site minimal maximal symmetry presented in [13], we embed the EW triplet $\Delta$ in the first $\mathrm{SO}(8)_{1}$ and put the singlet $\eta$ at the second site as a singlet of $\mathrm{SO}(8)_{2}$. On the second site, a heavy composite Dirac fermion $\Psi_{35}$ in the 35 (traceless symmetric) representation of the $\mathrm{SO}(8)_{2}$ gauge group is introduced, which will mix with the $\Delta$ (embedded in another 35 representation of the $\mathrm{SO}(8)_{1}$ global symmetry) and the $\eta$ fermions.

The most general Lagrangian for these fermions invariant under the global $\mathrm{SO}(8)_{1} \times \mathrm{SO}(8)_{2}$ is given by

$$
\begin{aligned}
\mathcal{L}_{\Delta \eta}= & \operatorname{Tr}\left[\bar{\Delta}\left(i \not D-M_{\Delta}\right) \Delta\right]+\bar{\eta}\left(i \not D-M_{\eta}\right) \eta \\
& +\operatorname{Tr}\left[\bar{\Psi}_{35}\left(i \not D-M_{35}\right) \Psi_{35}\right]-\left\{\lambda_{\Delta_{L}} \operatorname{Tr}\left[\bar{\Psi}_{\Delta_{L}} U_{1} \Psi_{35_{R}} U_{1}^{T}\right]\right. \\
& \left.+\lambda_{\eta_{L}} \mathcal{H}^{\prime \dagger} \bar{\Psi}_{35_{R}} \mathcal{H}^{\prime} \eta_{L}+(L \leftrightarrow R)+\text { H.c. }\right\} .
\end{aligned}
$$

The composite sector has a chiral $\mathrm{SO}(8)_{2 L} \times \mathrm{SO}(8)_{2 R}$ global symmetry in the $M_{35} \rightarrow 0$ limit, which is broken by the fermion mass leaving behind the $\mathrm{SO}(8)_{2 V}$ maximal symmetry. In the $\lambda_{\Delta} \rightarrow 0$ limit this $\mathrm{SO}(8)_{2 V}$ symmetry can be identified with the global shift symmetry of NGBs in $\mathcal{H}^{\prime}$, while in the $\lambda_{\eta} \rightarrow 0$ limit with the shift symmetry of $U_{1}$. Thus, we can see that the global shift symmetry is collectively broken and the Higgs potential must be proportional to $\left(\lambda_{\eta} \lambda_{\Delta}\right)^{2}$. This collective nature of the breaking will also ensure that the Higgs potential is at most logarithmically divergent. Upon integrating out the massive $\Psi_{35}$, only the kinetic and mass mixing terms between $\Delta$ and $\eta$ will depend on the Higgs due to maximal symmetry

$$
\begin{aligned}
\mathcal{L}_{\text {eff }}= & \Pi_{L}^{1} \mathcal{H}^{\dagger} \bar{\Psi}_{\Delta_{L}} \mathcal{H} \not p \eta_{L}+M_{L}^{\Delta \eta} \mathcal{H} \dagger \bar{\Psi}_{\Delta_{R}} \mathcal{H} \eta_{L} \\
& +(L \leftrightarrow R)+\text { H.c. }
\end{aligned}
$$

where $\Pi_{L, R}^{1}$ and $M_{L, R}^{\Delta \eta}$ are form factors resulting from integrating out of $\Psi_{35}$. $\mathcal{H} \equiv U_{1} \mathcal{H}^{\prime}$ contains the uneaten NGBs which can be parametrized in unitary gauge as $\mathcal{H}=\left(0,0,0, s_{h}, 0,0,0, c_{h}\right)$, with $s_{h} \equiv \sin (h / f) \quad$ and $c_{h} \equiv \cos (h / f)$. If we express this effective Lagrangian in terms of the Higgs field, we find that all mixing terms are proportional to $s_{h}^{2}$, as expected from our general discussion at the beginning of this Letter. Thus, the loop-induced Higgs potential contains only quartic terms (or higher) in the Higgs field. For $s_{h} \ll 1$, expanding the Higgs potential to leading term, we indeed get $V_{f} \approx \beta_{\Delta} s_{h}^{4}$.

Note that in the most general case, in addition to the kinetic mixing terms giving rise to a positive shift in the quartic we also obtain momentum independent left-right mixing terms in the effective Lagrangian (7). These will generate a negative contribution to the Higgs quartic coupling as we discussed earlier. In total there are four independent triplet-singlet mixing terms in the effective Lagrangian and any two of them can be contracted in a loop to give contributions to the Higgs quartic coupling. The sum of these contributions can be both positive and negative: the sign depends on the actual choices of the parameters in the model. So in a realistic model, $\beta_{\Delta}$ can be positive or negative. We uniformly scanned the whole parameter space and found that the region corresponding to a positive $\beta_{\Delta}$ is large (see Fig. 1), which implies that in this model we can naturally obtain a positive shift Higgs for the quartic coupling.

Our mechanism of inducing a positive quartic is particularly interesting in the context of THMs because the $Z_{2}$ parity $s_{h} \leftrightarrow c_{h}$ [10] between the SM and twin sector has to be broken to achieve realistic EWSB, which usually reintroduces some sensitivity to the partner masses and some of the tuning in ordinary THMs [17-19]. The beauty of our mechanism of generating the quartic is that this could be the sole source of $Z_{2}$ breaking, allowing the top and gauge sectors to remain exactly $Z_{2}$ invariant and without introducing any tuning. In essence the Higgs quartic mechanism will ensure that the $Z_{2}$ symmetry of the gauge and top sectors has the maximal effect on softening the Higgs potential [22].

The construction of a $Z_{2}$ invariant top and gauge sector is identical to the case of the ordinary $\mathrm{SO}(8) / \mathrm{SO}(7)$ twin 
Higgs models. The version with minimal maximal symmetry can be obtained by adding the left handed top doublet and the twin top doublet at the first site and the right handed top as well as the twin top at the second site as a singlets of $\mathrm{SO}(8)_{2}$, just like in the construction in [13]. The one-loop induced Higgs potential from the top and gauge sectors appear at $\mathcal{O}\left(y_{t}^{2}\right), \mathcal{O}\left(g^{2}\right)$ and will be canceled by their twin partners due to the $Z_{2}$ parity. Thus, the leading order Higgs potential is at $\mathcal{O}\left(y_{t}^{4}\right), \mathcal{O}\left(g^{4}\right)$ of the form

$$
V(h) \approx\left(\beta_{f}-\beta_{g}\right)\left(s_{h}^{4}+c_{h}^{4}\right)+\beta_{\Delta} s_{h}^{4},
$$

where we have added the extra quartic contribution from our mechanism and $\beta_{f, g}$ is from the $Z_{2}$ preserving top and gauge contributions which can be parametrized as

$$
\begin{aligned}
& \beta_{f} \simeq c_{f}^{\prime} \frac{N_{c} y_{t}^{4} f^{4}}{(4 \pi)^{2}} \ln \frac{M_{f}^{2}}{m_{t}^{2}}, \quad \beta_{g} \simeq c_{g}^{\prime} \frac{9 f^{4} g^{4}}{1024 \pi^{2}} \ln \frac{m_{\rho}^{2}}{m_{W}^{2}}, \\
& \beta_{\Delta} \simeq c_{\Delta \eta}^{\prime} \frac{\left(\lambda_{\Delta} \lambda_{\eta}\right)^{2}}{(4 \pi)^{2}} \ln \frac{\Lambda^{2}}{M_{i}^{2}},
\end{aligned}
$$

where $M_{f}$ is a typical mass scale of the top partners, $m_{\rho}$ is the gauge resonance mass, $M_{i}$ is the mass of $\Delta$ or $\eta, \Lambda \sim$ $4 \pi f$ is the cutoff in the composite sector, $\lambda_{\Delta, \eta}=\left\{\lambda_{\Delta_{L}, \eta_{L}}\right.$, $\left.\lambda_{\Delta_{R}, \eta_{R}}\right\}$, and $c_{f, g, \Delta \eta}^{\prime}$ are $\mathcal{O}(1)$ numerical parameters. Since the quadratic divergences are canceled by the twin partners both in the gauge and top sectors hence $\beta_{g, f}$ are only logarithmically sensitive to the mass of the vector boson and colored top partners. Thus, the Higgs can be light even for heavy colored top partners with only a mild logarithmic tuning.

Finally, we discuss the EWSB of the Higgs potential and show that the twin Higgs model with our mechanism for generating the Higgs quartic can give rise to a realistic minimum with no tuning at all.

In composite Higgs models the Higgs potential can be parametrized as

$$
V(h)=-\left(\gamma_{f}-\gamma_{g}\right) s_{h}^{2}+\beta s_{h}^{4},
$$

where it is assumed that for realistic EWSB vacuum expectation value (VEV) $s_{h} \ll 1$ hence higher powers of $s_{h}$ are neglected and $\gamma_{g}$ and $\gamma_{f}$ are the contributions from the gauge and fermion sectors. In our model, from Eq. (8), $\gamma_{g, f}=2 \beta_{g, f}, \beta=2\left(\beta_{f}-\beta_{g}\right)+\beta_{\Delta}$. The overall $\beta$ has to be positive for a realistic model so the PNGB Higgs will acquire a VEV if $\gamma_{f}-\gamma_{g} \equiv \gamma>0$ with a minimum at

$$
\xi \equiv s_{h}^{2}=\frac{\gamma}{2 \beta}=\frac{\beta_{f}-\beta_{g}}{\beta},
$$

where $\xi$ is a parameter measuring the separation between $f$ and EWSB scale $v$. The Higgs mass in this vacuum is $m_{h}^{2}=8 \beta \xi(1-\xi) / f^{2}$, and we can see that for a fixed value $f, \beta$ is also fixed. Using $v^{2} / f^{2} \approx \xi$, we see that the Higgs mass depends only on $\beta / f^{4}$. The value of $\beta$ reproducing $m_{h}=125 \mathrm{GeV}$ is

$$
\frac{\beta}{f^{4}}=\frac{m_{h}^{2}}{8 v^{2}(1-\xi)}=\frac{0.032}{1-\xi} .
$$

Moreover, from Eq. (8), we can see that $\beta_{\Delta} / f^{4}=$ $0.032(1-2 \xi) /(1-\xi)$.

Before quantifying the tuning we first illustrate that our mechanism can indeed produce a sufficiently large positive Higgs quartic coupling. To demonstrate that we can easily achieve the value $\beta$ from (12) we show a contour plot of $\beta_{\Delta} / f^{4}$ in part of the right-handed coupling parameter space in Fig. 1 while fixing other parameters in natural values. We see that for a sizable fraction of the parameter space $\beta_{\Delta}$ is sufficiently large to produce the observed Higgs mass.

One important feature of our model is that it naturally predicts $\xi$ around 0.15 with $M_{f}>2 \mathrm{TeV}$ and $m_{\rho}>4 \mathrm{TeV}$ and no tuning $(\Delta \sim 1)$, because $\beta_{f}$ and $\beta_{g}$ only have a logarithmical dependence on $M_{f}$ and $m_{\rho}$ from Eq. (9). Indeed, together with Eqs. (11) and (12), we have

$$
\xi=\frac{\beta_{f}-\beta_{g}}{\beta} \approx \frac{\beta_{f}}{\beta} \simeq c_{f}^{\prime} \frac{N_{c} y_{t}^{2}}{2 \pi^{2}}\left(\frac{m_{t}}{m_{h}}\right)^{2} \ln \left(\frac{M_{f}^{2}}{m_{t}^{2}}\right) .
$$

One can clearly see that the predicted small value of $\xi$ around 0.15 is a consequence of one-loop suppression together with the logarithmic enhancement by the colored top partner mass $M_{f}$, in addition to the numerical factors $N_{c}, c_{f}^{\prime}$.

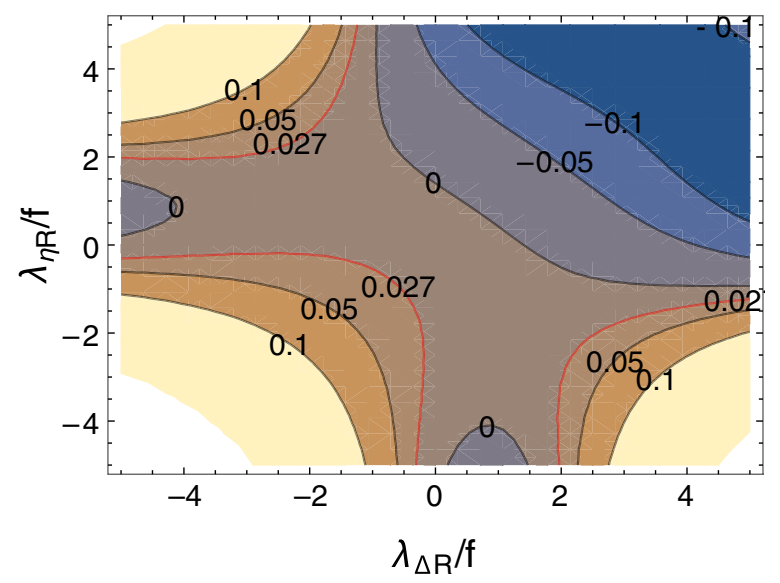

FIG. 1. Contour plot for the Higgs quartic coupling $\beta_{\Delta} / f^{4}$ ( $\xi$ fixed at 0.15 ) as a function of the right-handed couplings $\lambda_{\Delta_{R}} / f$ and $\lambda_{\eta_{R}} / f$ with the masses of the triplet, the singlet and the $\Psi_{35}$ multiplets fixed at $4 \mathrm{TeV}, M_{\Delta}=M_{\eta}=M_{35}=4 \mathrm{TeV}$, and the left-handed couplings fixed at $\lambda_{\Delta_{L}}=2 f, \lambda_{\eta_{L}}=2 f$. The red line with $\beta_{\Delta} / f^{4} \approx 0.0266$ correspond to physical Higgs mass $m_{h}=125 \mathrm{GeV}$. 
The main source of tuning in ordinary THMs is the sensitivity to $m_{\rho}$. It remains minimal $\sim 1 / \xi$ when the $\rho$ meson is light $\left(m_{\rho}<2.25 \mathrm{TeV}\right.$ for $\left.\xi=0.15\right)$ and will grow linearly with $m_{\rho}^{2}$ for heavy $m_{\rho}$. For the current bound $m_{\rho}>$ $3 \mathrm{TeV}$ [23], this corresponds to $\Delta^{\mathrm{TH}}>12$. Therefore, we can see that our mechanism substantially helps the tuning of the Higgs potential.

In our model, smaller values of $\xi$ can be easily obtained by turning on $Z_{2}$ breaking contributions from the hypercharge $U(1)_{Y}$ sector (or light quark sector). Thus, the gauge contributions will be enhanced and quadratically sensitive to $m_{\rho}$. Using this we can require a mild cancellation between the enhanced $\gamma_{g}$ and $\gamma_{f}$ as in ordinary twin Higgs models $[18,19]$ and meanwhile enhance $\beta_{\Delta}$ to achieve smaller $\xi$, which will result in a small tuning of about $\Delta \sim 3$ for $\xi=0.1$ with $M_{f} \in[2,2.5] \mathrm{TeV}$ and $m_{\rho} \in[3,3.5] \mathrm{TeV}$. The natural value $\xi=0.15$ of our simplest model is consistent with the current LHC bound $\xi<0.2$ [24]. However, future lepton colliders like CEPC, ILC, or TLEP will be able to test $\xi$ down to much smaller values.

An adjustable Higgs quartic self-coupling can play an important role in producing a natural Higgs potential and solving the little hierarchy problem. In this work we proposed a novel mechanism for producing such an adjustable Higgs quartic term. It is based on the observation that a kinetic mixing between EW singlet and triplet fermions can result in a positive contribution to the Higgs quartic. This mechanism is very simple and can be implemented in any composite PNGB Higgs model. We presented an explicit realization in a two site composite twin Higgs model with the minimal implementation of maximal symmetry. In twin Higgs models the extra Higgs quartic can be used as the (only) source for $Z_{2}$ breaking while keeping both the gauge and top sectors $Z_{2}$ invariant. As a result the Higgs potential will be largely insensitive to colored top and gauge partner masses. These twin Higgs models will have a fully natural EWSB sector with the heavy colored partners outside of the LHC direct detection bounds.

C. C. thanks the Technical University of Munich for a fruitful visit while working on this project supported by a research prize by the Humboldt Research Foundation. T. M. thanks the Cornell Particle Theory group for its hospitality while working on this project. C. C. is supported in part by the NSF Grant No. PHY-1719877 as well as the BSF Grant No. 2016153. J. S. is supported by the National Natural Science Foundation of China (NSFC) under Grants No. 11947302, No. 11690022, No. 11851302, No. 11675243, and No. 11761141011, and also supported by the Strategic Priority Research Program of the Chinese Academy of Sciences under Grants No. XDB21010200 and No. XDB23000000. T. M. is supported in part by Project No. Y6Y2581B11 supported by 2016 National Postdoctoral Program for Innovative Talents.
*Corresponding authors. jshu@itp.ac.cn t.ma@campus.technion.ac.il

[1] D. B. Kaplan and H. Georgi, Phys. Lett. 136B, 183 (1984).

[2] H. Georgi and D. B. Kaplan, Phys. Lett. 145B, 216 (1984).

[3] M. J. Dugan, H. Georgi, and D. B. Kaplan, Nucl. Phys. B254, 299 (1985).

[4] N. Arkani-Hamed, A. G. Cohen, E. Katz, and A. E. Nelson, J. High Energy Phys. 07 (2002) 034; N. Arkani-Hamed, A. G. Cohen, E. Katz, A. E. Nelson, T. Gregoire, and J. G. Wacker, J. High Energy Phys. 08 (2002) 021.

[5] K. Agashe, R. Contino, and A. Pomarol, Nucl. Phys. B719, 165 (2005).

[6] R. Contino, arXiv:1005.4269.

[7] B. Bellazzini, C. Csáki, and J. Serra, Eur. Phys. J. C 74, 2766 (2014).

[8] G. Panico and A. Wulzer, Lect. Notes Phys. 913, 1 (2016).

[9] M. Schmaltz and D. Tucker-Smith, Annu. Rev. Nucl. Part. Sci. 55, 229 (2005).

[10] Z. Chacko, H. S. Goh, and R. Harnik, Phys. Rev. Lett. 96, 231802 (2006).

[11] C. Csáki, T. Ma, and J. Shu, Phys. Rev. Lett. 121, 231801 (2018).

[12] C. Csáki, T. Ma, and J. Shu, Phys. Rev. Lett. 119, 131803 (2017).

[13] C. Csáki, T. Ma, J. Shu, and J. H. Yu, arXiv:1810.07704.

[14] C. Csáki, M. Geller, and O. Telem, J. High Energy Phys. 05 (2018) 134.

[15] N. Arkani-Hamed, A. G. Cohen, and H. Georgi, Phys. Lett. B 513, 232 (2001).

[16] This could be enforced by imposing a chiral $Z_{2}$ parity with charges $\eta_{L}(-), \eta_{R}(+), \Delta_{L}(-), \Delta_{R}(+), H(+), \Psi_{35_{L, R}}(-)$. The $\Delta$ and $\eta$ masses would have to be generated by an electroweak singlet scalar whose VEV spontaneously breaks this parity. However, such scalar VEV could lead to a domain wall problem, and one would still have to explain why $\lambda_{1,2 R}$ are not generated via the insertion of the singlet scalar VEV.

[17] M. Geller and O. Telem, Phys. Rev. Lett. 114, 191801 (2015); C. Csáki, M. Geller, O. Telem, and A. Weiler, J. High Energy Phys. 09 (2016) 146.

[18] M. Low, A. Tesi, and L. T. Wang, Phys. Rev. D 91, 095012 (2015).

[19] R. Barbieri, D. Greco, R. Rattazzi, and A. Wulzer, J. High Energy Phys. 08 (2015) 161.

[20] Z. Chacko, H. S. Goh, and R. Harnik, J. High Energy Phys. 01 (2006) 108; G. Burdman, Z. Chacko, H. S. Goh, and R. Harnik, J. High Energy Phys. 02 (2007) 009.

[21] N. Craig, S. Knapen, and P. Longhi, Phys. Rev. Lett. 114, 061803 (2015).

[22] This $Z_{2}$ symmetry will be broken by the RGE running of the $\mathrm{SU}(2)_{L}$ coupling due to the electroweak triplet $\Delta$. This $Z_{2}$ breaking is considerably smaller than in the ordinary twin Higgs model and can be neglected.

[23] G. Aad et al. (ATLAS Collaboration), J. High Energy Phys. 09 (2019) 091.

[24] J. de Blas, O. Eberhardt, and C. Krause, J. High Energy Phys. 07 (2018) 048. 